\title{
Clinical Progress Note: Goal-Directed N-acetylcysteine Treatment of Acetaminophen Toxicity
}

\author{
Brad Hall, PharmD, MS ${ }^{1 *}$, Kayla Wilson, PharmD, MS², Fred Blind, MD²
}

'University of South Florida Morsani College of Medicine, Tampa, Florida; ${ }^{2}$ Lakeland Regional Health, Lakeland, Florida.

\section{A} ccording to the most recent Annual Report of the American Association of Poison Control Centers' National Poison Data System, there were 51,081 patient exposures to acetaminophen or acetaminophen-containing products treated in a healthcare facility in 2018. ${ }^{1}$ The toxicity of acetaminophen is mediated by its metabolism into the electrophile $\mathrm{N}$-acetyl-p-benzoquinone imine (NAPQI). ${ }^{2}$ If the amount of acetaminophen ingested is higher than recommended dosages, glutathione stores may become depleted as the liver tries to detoxify NAPOI and may no longer able to keep up with the demand. As the patient progresses 24 to 36 hours after ingestion, the onset of hepatic injury becomes apparent with elevations in aspartate aminotransferase (AST). AST elevations, should they occur, are almost always present by 36 hours. ${ }^{2}$ Maximum hepatic dysfunction, as well as elevations in prothrombin time, bilirubin, and serum creatinine, occurs 72 to 96 hours after a toxic ingestion. As a result of marked age-associated differences in the process of conjugating acetaminophen metabolites, infants and young children may be less susceptible to acetaminophen-associated hepatotoxicity, compared with their adult counterparts. ${ }^{3,4}$

This clinical progress note addresses acetaminophen toxicity treatment, focusing on $\mathrm{N}$-acetylcysteine (NAC) dosing protocols and the 2017 American College of Medical Toxicology (ACMT) position statement, Duration of Intravenous Acetylcysteine Therapy Following Acetaminophen Overdose. ${ }^{5}$ We conducted a literature search via the PubMed database. The authors began by using the Medical Subject Headings terms "acetaminophen overdose [title]," which yielded 299 articles, "acetaminophen hepatotoxicity [title]," which yielded 283 articles, and "acetaminophen N-acetylcysteine [title]," which yielded 335 titles. Variations of these terms were used to ensure exhaustive search results. The search results were reviewed for applicability to acetaminophen poisoning and especially goal-directed NAC treatment strategies.

\section{INITIAL TREATMENT}

Clarifying a patient's history of ingestion is a crucial first step to the diagnosis and risk evaluation of acetaminophen-poisoned

*Corresponding Author: Brad Hall, PharmD, MS; Email: abhall2@usf.edu; Telephone: 863-687-1100 ext. 2728.

Published online first August 20, 2020.

Received: October 15, 2019; Revised: April 14, 2020; Accepted: April 15, 2020

(c) 2021 Society of Hospital Medicine DOI 10.12788/jhm.3447 patients. The initial laboratory workup should include, at minimum, measurement (at 4 or more hours after ingestion) of serum acetaminophen level, AST and alanine aminotransferase $(A L T)$, bilirubin, alkaline phosphatase, creatinine, prothrombin time and international normalized ratio (PT/INR), lactate, and ethanol levels. Gastric decontamination is not appropriate in most cases because of the rapid absorption of acetaminophen. ${ }^{2}$ Activated charcoal is most effective when given within the first 2 hours following ingestion. For acetaminophen-poisoned patients with severe hepatic toxicity, treating clinicians should consider consultation with a medical toxicologist, an individual with expertise, or the local Poison Control Center. ${ }^{5}$

The Rumack-Matthew nomogram provides guidance on the initiation of antidotal treatment for patients with acetaminophen overdose. This nomogram was developed to exhibit high sensitivity in patients at risk for hepatic damage from acetaminophen overdose. ${ }^{6}$ The mainstay for treatment of acetaminophen poisonings is NAC. Early administration of NAC can replenish depleted hepatic glutathione stores, which restores the liver's ability to detoxify the toxic metabolite NAPQI. When administered within 6 to 8 hours following ingestion, NAC is nearly universally effective at preventing the subsequent hepatic damage from NAPQI. ${ }^{7}$ Consideration of individualized factors should lead the treating clinician to use the Rumack-Matthew nomogram within the appropriate contexts. Factors such as an unreliable timeline of ingestion by patients, concomitantly ingested medications, and patient-specific factors that might alter the absorption of acetaminophen or impact serum acetaminophen concentrations should be considered. While this nomogram is helpful for decision-making pertaining to the initiation of NAC, further management and continuation of treatment must be patient specific and determined by the treating clinician.

In the United States, intravenous and oral formulations of NAC are available for treatment of acetaminophen toxicity. These formulations are equally efficacious in treating acetaminophen toxicity, with the exception of established hepatic failure, for which only the intravenous route has been studied. ${ }^{8}$ Oral administration results in vomiting in approximately $20 \%$ of patients, while intravenous administration may uncommonly result in severe anaphylactoid reactions. Three scenarios exist in which the intravenous route is preferred over the oral route: acetaminophen toxicity in pregnant women, acetaminopheninduced hepatic failure, and intractable vomiting preventing treatment with oral therapy. ${ }^{2}$ The intravenous formulation is Food and Drug Administration-approved for a 21-hour 
treatment duration, while the oral formulation is traditionally administered for a 72-hour duration. 9.10

\section{LIMITATIONS OF CURRENT TREATMENT ALGORITHMS}

Treatment of acetaminophen poisoning must be tailored on an individual basis and should not follow predetermined durations of treatment. Not only might the FDA-approved 21-hour course of intravenous NAC be inadequate, but the standard 72-hour oral regimen duration may be excessive for many patients. Both the 21-hour and 72-hour protocols were developed based on the half-life and expected elimination of acetaminophen from the body. The traditional 72-hour oral protocol used in the United States was approved in 1985 and was based on the observation that, in patients who developed acetaminophen-induced fatal hepatotoxicity, the acetaminophen half-life was 12 hours, requiring five half-lives to eliminate the drug, for a total of 60 hours. ${ }^{11}$ The FDA then added an additional 12 hours to provide an additional margin of safety. Researchers in the United Kingdom developed an intravenous treatment protocol based on a shorter 4-hour acetaminophen half-life, calculated from observations of a larger pool of acetaminophen-poisoned patients and not just those with fatal hepatotoxicity. ${ }^{12}$ This 21 -hour protocol-slightly longer than the five 4-hour half-lives needed-was subsequently approved in the United States as the intravenous formulation's duration in 2004.

Case reports of hepatic failure have described treatment failures of intravenous NAC after the completion of the standard 21 hour treatment regimen. ${ }^{13} \mathrm{~A}$ case report by Smith et al describes a patient with significant single ingestion who ultimately developed hepatotoxicity despite receiving the FDA-approved 21-hour infusion. ${ }^{13}$ This patient ingested approximately 96 immediate-release, 500-mg acetaminophen tablets over approximately 1 hour. The initial acetaminophen concentration was $264 \mu \mathrm{g} / \mathrm{mL}$ at 2.25 hours after ingestion. At 21 hours after ingestion, the patient's acetaminophen levels remained elevated at $116 \mu \mathrm{g} / \mathrm{mL}$, but therapy with NAC was discontinued per protocol because AST and ALT levels were normal. A second peak in serum acetaminophen levels occurred after discontinuation of NAC, with levels reaching $228 \mu \mathrm{g} / \mathrm{mL}$ approximately 48 hours after ingestion. Liver function worsened over the next few days, with elevations in AST of greater than 4,000 U/L, PT of 51.4 seconds, and ammonia of $165 \mu \mathrm{g} / \mathrm{dL}$. Intravenous NAC was restarted and hepatic function improved gradually. The patient was ultimately discharged from the hospital. This case and others highlight the possible pitfalls of applying a protocolized, fixed treatment duration of NAC to all patients.

\section{GOAL-DIRECTED TREATMENT RECOMMENDATIONS}

Based on individual ingestion timing, quantities, and pharmacokinetics, patients should have pertinent laboratory markers monitored at least every 24 hours from presentation. The clinician may then consider the need to continue or discontinue NAC treatment every 24 hours from the initiation of NAC. Although notable limitations exist for both intravenous and oral treatment regimens, the literature uniformly recommends treating until serum acetaminophen levels are undetectable, regardless of NAC administration route. Based on the metabolism of acetaminophen, as long as serum concentrations of acetaminophen persist, further metabolism to the toxic NAPQI metabolite is possible. The ACMT addressed these concerns with the 2017 position paper, which strongly recommends all of the following criteria to be present prior to discontinuation of intravenous NAC: undetectable acetaminophen concentration, improving hepatic aminotransferases, and improving prognostic markers, including creatinine, lactate, $\mathrm{pH}$, PT/INR, and phosphate. ${ }^{5}$ In cases in which laboratory and clinical parameters are abnormal, continued treatment beyond the established standard NAC treatment durations is warranted. However, once NAC treatment is extended beyond established protocol lengths, the subsequent dosing regimens that should be employed in these patients are not well studied. The ACMT Position Statement states the administration of an additional NAC bolus or extending the duration of the $6.25 \mathrm{mg} / \mathrm{kg}$ per hour maintenance infusion may be appropriate in this subset of patients requiring continued treatment.

Based on the positive outcomes in most patients receiving the 21-hour intravenous NAC protocol, the necessity of an additional 2 days of NAC treatment with the 72-hour oral regimen is uncertain. The ACMT Position Statement states "evidence supports using shorter oral NAC courses, provided that liver enzymes and synthetic function are normal or improving, and plasma acetaminophen concentration is undetectable." Based on this and available literature, patients with a lower risk for developing hepatotoxicity may have oral NAC therapy discontinued after 24 to 48 hours of treatment if specific lab and patient parameters are within defined criteria.

The ACMT does not differentiate between adult and pediatric recommendations. However, the data used to develop the ACMT goal-directed treatment recommendations included both adult and pediatric patients. ${ }^{14,15}$ The same goal-directed treatment principles may be applied to pediatric patients, though studies have not specifically addressed pediatric goal-directed treatment of acetaminophen poisoning.

\section{CONCLUSION}

Acetaminophen-poisoned patients require evaluation not just prior to initiation of treatment, but also when deciding to discontinue NAC therapy. New literature supports reconsideration of standardized 21- and 72-hour NAC treatment protocols, as well as the notion that treatment duration should be guided by laboratory parameters rather than by route of NAC administration. Goal-directed or patient-tailored treatment is also supported by the ACMT. ${ }^{5}$ Treatment with NAC should continue in patients displaying hepatic toxicity or persistent elevations in acetaminophen levels. In these cases, the optimal treatment regimen remains unknown and further research is warranted.

Disclosures: The authors report no conflicts of interest. 


\section{References}

1. Gummin D, Mowry J, Spyker D, et al. 2018 Annual Report of the American Association of Poison Control Centers' National Poison Data System (NPDS): 36th Annual Report. Clin Toxicol (Phila). 2019;57(12):1220-1413. https://doi.org/10.1080/15563650.2019.1677022

2. Hoffman RS, Howland MA, Lewin NA, Nelson LS, Goldfrank LR, eds. Goldfrank's Toxicologic Emergencies, Tenth Edition. McGraw Hill Education; 2015.

3. Miller RP, Roberts RJ, Fischer LJ. Acetaminophen elimination kinetics in ne onates, children, and adults. Clin Pharmacol Ther. 1976;19:284-294. https:// doi.org/10.1002/cpt1976193284

4. Rumack $\mathrm{BH}$. Acetaminophen overdose in young children: treatment and effects of alcohol and other additional ingestants in 417 cases. Am J Dis Child. 1984;138(5):428-433. https://doi.org/10.1001/archpedi.1984.02140430006003

5. American College of Medical Toxicology. ACMT position statement: duration of intravenous acetylcysteine therapy following acetaminophen overdose. J Med Toxicol. 2017;13(1):126-127. https://doi.org/10.1007/s13181-016-0542-z

6. Matthew H. Acute acetaminophen poisoning. Clin Toxicol. 1973;6(1):9-11. https://doi.org/10.3109/15563657308991037

7. Smilkstein MJ, Knapp GL, Kulig KW, Rumack BH. Efficacy of oral N-acetylcysteine in the treatment of acetaminophen overdose: analysis of the national multicenter study (1976 to 1985). N Engl J Med. 1988;319(24):1557-1562. https://doi.org/10.1056/nejm198812153192401

8. Keays R, Harrison PM, Wendon JA, et al. Intravenous acetylcysteine in parac- etamol induced fulminant hepatic failure: a prospective controlled trial. BMJ. 1991;303(6809):1026-1029. https://doi.org/10.1136/bmj.303.6809.1026

9. Acetadote $®$ (acetylcysteine injection). Package Insert. Cumberland Pharmaceuticals; 2004.

10. Acetylcysteine Solution. Package insert. Roxanne Laboratories; 2007.

11. Wong A, Graudins A. Risk prediction of hepatotoxicity in paracetamol poisoning. Clin Toxicol (Phila). 2017;55(8):879-892. https://doi.org/10.1080/1556 3650.2017.1317349

12. Prescott LF. Kinetics and metabolism of paracetamol and phenacetin. Br J Clin Pharmacol. 1980;10(Suppl 2):291S-298S. https://doi.org/10.1111/ j.1365-2125.1980.tb01812.x

13. Smith SW, Howland MA, Hoffman RS, Nelson LS. Acetaminophen overdose with altered acetaminophen pharmacokinetics and hepatotoxicity associated with premature cessation of intravenous $\mathrm{N}$-acetylcysteine therapy. Ann Pharmacother. 2008;42(9):1333-1339. https://doi.org/10.1345/aph.1k680

14. Woo OF, Mueller PD, Olsen KR, Anderson B, Kim SY. Shorter duration of oral $\mathrm{N}$-Acetylcysteine therapy for acute acetaminophen overdose. Ann Emerg Med. 2000; 35(4):363-368. https://doi.org/10.1016/S0196-0644(00)70055-2

15. Betten D, Cantrell F, Thomas S, Williams S, Clark R. A prospective evaluation of shortened course oral $\mathrm{N}$-acetylcysteine for the treatment of acute acetaminophen poisoning. Ann Emerg Med. 2007;50(3):272-279. https://doi. org/10.1016/j.annemergmed.2006.11.010 